peptide, analogue de la vasopressine laisse penser que d'autres peptides de cette taille pourraient à leur tour être absorbés par la muqueuse intestinale et être actifs par voie orale.

Paul Czernichow

Unité d'Endocrinologie Pédiatrique et Diabète. Hôpital des Enfants-Malades. 149, rue de Sèves, 75743 Paris Cedex 15.

\section{Analogues de la LHRH et fonction gonadotrope}

La structure de la gonadolibérine (LHRH ou GNRH) a été élucidée en 1971. Depuis lors, de nombreux analogues à action prolongée, dits agonistes, ont été étudiés in vitro et pour certains au stade clinique [13]. Les premières hypothèses prévoyant une arme thérapeutique se substituant à la LHRH endogène n'ont pas été totalement confirmées. En effet les travaux de Knobil ont montré que l'administration continue de LHRH ne permettrait pas de restaurer la physiologie normale chez le singe rendu hypogonadotropique par lésions du noyau arqué [14]. Une administration discontinue, reproduisant la séquence de pulsatilité normale était par contre efficace. La même observation a été faite chez l'homme en utilisant un agoniste à faible dose et de façon discontinue.

La perfusion continue de LHRH, ou l'administration à intervalles rapprochés d'un agoniste lentement métabolisé conduisent en revanche à un blocage de la fonction gonadotrope endogène. De ces constatations naissait le concept de la désensibilisation des cellules gonadotropes. Celle-ci est due, au moins en partie, à l'occupation prolongée des récepteurs de la LHRH qui empêche le maintien d'une fonction gonadotrope pulsatile normale. Ainsi apparaît la possibilité de traiter les enfants atteints de puberté précoce, en provoquant une inhibition de la fonction gonadique durable mais surtout réversible. Plusieurs types de peptides sont actuellement utilisés : certains decapeptides représentent les séquences de la LHRH avec substitution d'acides aminés (telle la D-Trp ${ }^{6}$ LHRH); d'autres sont des LHRH (1-9) nonapeptides éthylamide différentes de la LHRH naturelle, par leur séquence $C$ terminale du type Pro 9 -éthylamide. En pratique, ces molécules ont un même mode d'action. Le degré d'affinité des récepteurs hypophysaires de la LHRH conditionne l'activité relative des analogues. Ces différences se reportent sur les doses nécessaires pour obtenir un effet thérapeutique. En clinique humaine, hormis ces différences de posologie, les résultats thérapeutiques sont similaires. Ceux-ci sont peut-être liés à la durée et à la permanence d'activité des peptides : une activité longue, obtenue au besoin par des véhicules retard, pouvant améliorer leur efficacité. Dans la puberté précoce d'origine centrale, le traitement par la LHRH apparaît maintenant comme le premier traitement efficace après les échecs partiels de la médroxyprogestérone antigonadotrope et de l'acétate de cyprotérone antiandrogène $[15,16]$. Le blocage complet de la progression pubertaire (croissance, maturation osseuse, sécrétion gonadique) est obtenu sans effets secondaires ni intolérance. La crainte de provoquer des lésions testiculaires chez le garçon ne semble plus fondée car à la différence du rat, il n'a pas été trouvé de récepteurs de LHRH dans le testicule humain. Il reste à démontrer que la fertilité ultérieure de ces sujets est préservée, ce qui paraît très probable. Ce traitement hautement spécifique de la seule fonction de reproduction a magistralement confirmé que la puberté précoce idiopathique était liée à une activation prématurée du système LHRH-gonadotrophines, dans la majorité des cas. Il a aussi révélé des formes résistantes aux analogues de la LHRH qui s'avèrent maintenant être de fausses pubertés précoces, par activité gonadique primitivement ou secondairement autonomes [17] :les taux circulants de LH ne présentent pas la pulsatilité caractéristique d'une commande centrale, la testostérone ou l'œstradiol plasmatique fluctuent sans rythme particulier et restent élevés malgré de fortes doses de LHRH. Dans ces cas, le mécanisme de l'hyperplasie leydienne, souvent familiale, ou l'autonomie ovarienne, décrite surtout dans le cadre du syndrome de McCune Albright, reste tout à fait inconnu.

A l'opposé de la puberté précoce, se situent les absences de puberté d'origine hypothalamique. Celles-ci sont, déjà à l'âge adulte, des indications de traitements à visée stimulatrice utilisant la délivrance périodique et durable de LHRH naturelle, afin de provoquer un état de maturation gonadique permettant une fertilité. Chez l'enfant, dans le cadre des hypogonadismes hypogonadotrophiques, on s'est toujours contenté du traitement androgénique ou œstrogénique substitutif classique. Des travaux récents montrent la possibilité de reproduire en quelques mois une séquence de développement somatique et de maturation gonadique identique à celle de la puberté spontanée. Il est possible que ce traitement par la LHRH doive être envisagé chez l'adolescent, malgré sa complexité technique, afin d'obtenir une meilleure maturation gonadique, à un âge où les implications psychologiques de cette maladie sont considérables. Ces perspectives thérapeutiques doivent encore être étudiées. Reste que le médecin dispose d'une arme thérapeutique lui permettant sans doute de contrôler le développement pubertaire dans diverses situations. Il importe d'en faire un dosage prudent et bien défini, respectant le développement spontané normal et ses grandes variations

\section{Raja Brauner Assistant des universités. Assis- tant des hôpitaux. \\ Raphaël Rappaport \\ Directeur de l'Unité Inserm U.30. Hôpital des Enfants-Ma- lades. 149, rue de Sères. 75743 Paris Cedex 15.}

